\title{
SOX19b regulates the premature neuronal differentiation of neural stem cells through EZH2-mediated histone methylation in neural tube development of zebrafish
}

Xian $\mathrm{Li}^{1,2+}$, Wenjuan Zhou ${ }^{1 \dagger}$, Xinyue $\mathrm{Li}^{1}$, Ming Gao ${ }^{1,3}$, Shufang $\mathrm{Ji}^{1}$, Wenyu Tian ${ }^{1}$, Guangyu $\mathrm{Ji}^{1}$, Jingyi Du ${ }^{1}$ and Aijun $\mathrm{HaO}^{1 *}$

\begin{abstract}
Objective: Neural tube defects (NTDs) are the most serious and common birth defects in the clinic. The SRY-related HMG box B1 (SoxB1) gene family has been implicated in different processes of early embryogenesis. Sox 19b is a maternally expressed gene in the SoxB1 family that is found in the region of the presumptive central nervous system (CNS), but its role and mechanism in embryonic neural stem cells (NSCs) during neural tube development have not yet been explored. Considering that Sox19b is specific to bony fish, we intended to investigate the role and mechanism of Sox19b in neural tube development in zebrafish embryos.

Material and methods: Morpholino (MO) antisense oligonucleotides were used to construct a Sox19b loss-offunction zebrafish model. The phenotype and the expression of related genes were analysed by in situ hybridization and immunolabelling. Epigenetic modifications were detected by western blot and chromatin immunoprecipitation.

Results: In this study, we found that zebrafish embryos exhibited a reduced or even deleted forebrain phenotype after the expression of the Sox19b gene was inhibited. Moreover, we found for the first time that knockdown of Sox19b reduced the proliferation of NSCs; increased the transcription levels of Ngn1, Ascl1, HuC, Islet1, and cyclindependent kinase (CDK) inhibitors; and led to premature differentiation of NSCs. Finally, we found that knockdown of Sox $19 \mathrm{~b}$ decreased the levels of EZH2/H3K27me3 and decreased the level of H3K27me3 at the promoters of Ngn 1 and asclia.

Conclusion: Together, our data demonstrate that Sox19b plays an essential role in early NSC proliferation and differentiation through EZH2-mediated histone methylation in neural tube development. This study established the role of transcription factor Sox $19 \mathrm{~b}$ and epigenetic factor EZH2 regulatory network on NSC development, which provides new clues and theoretical guidance for the clinical treatment of neural tube defects.
\end{abstract}

Keywords: Zebrafish, Neural tube defects, Sox19b, Neural stem cells

\footnotetext{
* Correspondence: aijunhao@sdu.edu.cn

${ }^{+}$Xian Li and Wenjuan Zhou contributed equally to this work.

'Key Laboratory of the Ministry of Education for Experimental Teratology, Shandong Provincial Key Laboratory of Mental Disorders, Department of Human Anatomy and Histoembryology, School of Basic Medical Sciences, Shandong University, 44\#, Wenhua Xi Road, Jinan 250012, Shandong, China Full list of author information is available at the end of the article
}

(c) The Author(s). 2019 Open Access This article is distributed under the terms of the Creative Commons Attribution 4.0 International License (http://creativecommons.org/licenses/by/4.0/), which permits unrestricted use, distribution, and reproduction in any medium, provided you give appropriate credit to the original author(s) and the source, provide a link to the Creative Commons license, and indicate if changes were made. The Creative Commons Public Domain Dedication waiver (http://creativecommons.org/publicdomain/zero/1.0/) applies to the data made available in this article, unless otherwise stated. 


\section{Background}

The embryonic neural tube is the precursor of the central nervous system. Neural tube development is a continuous development process from the appearance of the nerve plate to the closure of the nerve tube [1]. In this process, congenital neurodevelopmental diseases caused by neural tube formation and closure disorder are called neural tube defects (NTDs) [2, 3]. NTDs are the most serious and common birth defects in clinical practice and are the main causes of disability and death in newborns $[4,5]$. Previous studies have shown that the pathogenesis of NTDs is mainly the result of genetic factors, environmental factors, and maternal factors. The clinical manifestations of NTDs include brain deformity, cerebral dysplasia, encephalocele, spina bifida, and spinal meningocele [6-8]. However, the molecular mechanisms of NTDs are not yet fully understood.

The proliferation, differentiation, and migration of neural stem cells (NSCs) are the cellular basis of neural tube development. An increasing number of studies have shown that exploring the regulatory mechanism of proliferation and differentiation of NSCs may be the key to revealing the pathogenesis of NTDs [9-12]. The transition of NSCs from proliferation to neuronal commitment is a multi-step process; pluripotent NSCs exit the cell cycle due to the dynamic interaction of extracellular and intracellular signals. Eventually, neurons with different cellular characteristics are produced [13, 14]. The SRY-related HMG box B1 (SoxB1) family is the most important transcription factor regulating the proliferation and differentiation of NSCs; the main SoxB1 family members are Sox1, Sox2, and Sox3 [15, 16]. Indeed, studies have shown that the SoxB1 family is closely related to NTDs. Loss or mutation of sox 2 in humans can cause hypoplasia of the hippocampus, corpus callosum, cortex, and hypothalamus; ventricular enlargement; and anophthalmia $[17,18]$. A new human study also found that in 75 patients with NTD, $16 \%$ had a complete loss of sox2 [19].

Zebrafish have the advantages of in vitro development, transparent embryo, and rapid development, which makes it a good animal model for studying the development of neural tube $[20,21]$. The SoxB1 family in zebrafish includes Sox1a, Sox1b, Sox2, Sox3, Sox19a, and Sox19b, where Sox19a and Sox19b are specific to bony fish $[22,23]$. The expression of Sox1a and Sox1b is not observed until the caudal bud stage is mainly expressed in the lens and is not related to the development of the neural tube. In our study, we focused on the other 4 SoxB1 family members. In combination with previous reports and our results, the expression characteristics of the SoxB1 family in zebrafish are as follows: only Sox19b was highly expressed during the maternal phase, Sox3 and Sox19a were expressed initially in the 1000 cell phase, and Sox2 was expressed at the beginning of the $30 \%$ epiboly phase. All 4 were expressed until 48 hpf. Sox2, Sox3, Sox19a, and Sox19b were expressed in the neuroectodermal region until the $75 \%$ of epiboly phase, but the expression of Sox 2 and Sox 3 changed as development progressed. Sox 2 is mainly expressed in the anterior part of the neural tube, optic vesicle, and retina, while Sox 3 is mainly expressed in the posterior brain, lens, and olfactory system; only Sox19a and Sox19b are expressed specifically throughout the neural tube [23]. However, the role of Sox19b in neural tube development has not been systematically investigated. The maternal expression of Sox19b and the specific neural tube distribution, which is similar to that of Sox 2 in mammals, attracted our attention.

In this study, we knocked down Sox19b in zebrafish embryos using morpholino (MO) antisense oligonucleotides and confirmed that Sox19b is functionally important in neural tube development. More importantly, we found that knockdown of Sox19b could lead to a decrease in the proliferation of NSCs in the neural tube and premature differentiation. In neural development, Sox19b is essential for the proper regulation of ngn1, ascl1, and her3. Moreover, in terms of mechanism, we found that Sox19b induces high levels of histone H3K27me3 through the activity of EZH2, maintains the levels of histone H3K27me3, promotes cell division and proliferation, and maintains the stem cell pool. In this study, the networks of transcription factors and epigenetic factors that regulate the fate of NSCs were established to deepen the understanding of NSC proliferation and differentiation. These findings indicate that Sox19b plays a central role in the development of neural tubes, and provide new ideas and clues for the clinical treatment of NTDs.

\section{Materials and methods \\ Zebrafish maintenance}

$\mathrm{AB}$ strains of zebrafish were maintained and bred according to standard procedures. The ESEN zebrafish culture system (ESEN EnvironScience, Beijing, China) was used to control the temperature and the day-night cycles. All embryos were fertilized and incubated at $28.5^{\circ} \mathrm{C}$ without crowding (five to ten embryos per millilitre).

\section{Microinjection of morpholino antisense oligonucleotides}

Two morpholine ring modified antisense oligonucleotides for Sox19b were designed in this study, and they targeted the $5^{\prime}$ untranslated region and the translation initiation region of Sox $19 \mathrm{~b}$ mRNA. The sequence is $5^{\prime}-$ TACATCATGCCACTTCTCGCTTTGA-3', 5'-GTCTT CAGCTCGTGCTCCATCATGC-3'. The standard control for experiments was the sequence 5'-GTGTTG 
AGGTCGTCCTCCATCATCC-3'. All oligonucleotides were diluted with distilled water and stored at $-20^{\circ} \mathrm{C}$. $\mathrm{MO}$ oligonucleotides were injected into zebrafish embryos at the one-cell stage with 5-9 ng/embryo, and the injection volume was generally $2-3 \mathrm{nl}$. The specificity and efficiency of the Sox19b MO oligonucleotides in zebrafish were determined after coinjection of a Sox19b MO or control MO with a GFP-tagged Sox19b mRNA (Fig. 2a). Zebrafish full-length Sox19b cDNA was amplified using 5 '-GCGGATCCATGATGTACAGCATGATGGA$3^{\prime}$ forward and 5'-GCTCTAGAGATGTGAGTGAGGG GAACAGTT-3' reverse primers, and the cDNA was cloned into pCS2-GFP expression vectors using BamHI and $\mathrm{XbaI}$ restriction sites.

\section{Reverse transcription polymerase chain reaction and quantitative real-time PCR}

Total RNA was extracted using an RNeasy kit (Qiagen, USA) according to the manufacturer's instructions. Total RNA was treated with DNase I. The cDNA was synthesized with a RevertAid ${ }^{\mathrm{m}}$ First Strand cDNA Synthesis Kit (Thermo Fisher Scientific) following the manufacturer's protocol. PCR was performed with EasyTaq PCR SuperMix (TransGen, Beijing, China). The protocol was performed as follows: initial denaturation at $95^{\circ} \mathrm{C}$ for $5 \mathrm{~min}$, then $95^{\circ} \mathrm{C}$ for $30 \mathrm{~s}, 56^{\circ} \mathrm{C}$ for $40 \mathrm{~s}, 72^{\circ} \mathrm{C}$ for $30 \mathrm{~s}$, repeated for 23 or 27 cycles (marked in Fig. 1a). The primer sequences were as follows: Sox19a (forward, 5'-catgtccatggtgaaaccag-3'; reverse, 5' -cgtaccggtgaggtaatgct-3'), Sox19b (forward, 5' -aaatatcctcttgcagcggg-3'; reverse, $5^{\prime}$-ctgttcatgtagggctgtgc-3'), Sox2 (forward, 5'-gaaccccaaaatgcacaattcg- $3^{\prime}$; reverse, $5^{\prime}$ acttgtccttcttcatcagggt- $3^{\prime}$ ), and Sox3 (forward, 5'-ccattccgcagtccaaca-3'; reverse, 5'-gattctcctgagccatcttc-3'). Realtime PCR was performed with SYBR Green Realtime PCR Master Mix (TOYOBO CO., Ltd., Japan). The primer sequences were as follows: $\mathrm{Ngn} 1$ (forward, $5^{\prime}$-cttcgctcacaactacatctgg-3'; reverse, 5'-cactacgtcggtttgcaagtat-3'), Hис (forward, 5'-taacggccctgtcattagca-3'; reverse, 5'-cgtgttgatagccttgtcgg-3'), ascl1a (forward, 5' -cgggtgaagcttgtgaacaa3'; reverse, 5' -ttttgggagatggtgggtga-3'), Her3 (forward, 5'tacacttcgacgaccacaca- $3^{\prime}$; reverse, $5^{\prime}$-tgaagtgtgtggtttctgcc$3^{\prime}$ ), actin (forward, 5'-tcttccagccttccttcctg-3'; reverse, 5'tggaaggagcaagagaggtg-3'), p21 (forward, $5^{\prime}$-gtgtcaggaaaagcagcaga-3'; reverse, $5^{\prime}$-gacgcttcttggcttggtag-3'), p27 (forward, $5^{\prime}$-ttcgcttgtctaatggcagc-3'; reverse, 5' -gtgcgtggaaaag tcgaagt-3'), p57 (forward, 5' -actttcctctcctcacccg-3'; reverse, $5^{\prime}$-aaatcgcctcccactcgtaa- $3^{\prime}$ ), EZH2 (forward, $5^{\prime}$-tgagaccaccagctcttcag-3'; reverse, $5^{\prime}$-ggtctttgtgccgatgagtc-3'), fgf3 (forward, 5'-cccaagggcgccttgtgccaggg-3'; reverse, 5'ccgtgttttaaagcccctcctgg-3'), fgf (forward, $5^{\prime}$-ttcacggttgagttatctattcc-3'; reverse, $5^{\prime}$-agtctttcccagaccatttttc- $\left.3^{\prime}\right)$, and er81 (forward, 5' -gaaaacttggggctccacgggct-3'; reverse, 5' gggaaggggatgctgggctctga- $\left.3^{\prime}\right)$. The expression of $\beta$-actin was used as an internal control, and the $2^{-\Delta \Delta C T}$ method was used to calculate the changes in the gene expression levels.

\section{In situ hybridization and immunolabelling}

Briefly, antisense RNA probes were synthesized with digoxigenin RNA labelling kits (Roche). Embryos were collected at different stages and were fixed in $4 \%$ paraformaldehyde. The fixed embryos were pre-treated with proteinase $\mathrm{K}$, washed with PBST, and then the egg membrane was removed. The embryos were prehybridized in $\mathrm{Hyb}^{-}$for $5 \mathrm{~min}$ and $\mathrm{Hyb}^{+}$for $4 \mathrm{~h}$ at $65^{\circ} \mathrm{C}$; then, they were incubated with antisense RNA probes $(1 \mathrm{ng} / \mu \mathrm{l})$ overnight. The following antisense RNA probes were used: Sox19a, Sox19b, otx2, pax2a, PCNA, Ngn1, Huc, and Islet 1 . The next day, the embryos were washed in washing liquid I, II, and III, and blocking solution was used to block the nonspecific binding sites. Then, the embryos were incubated with anti-DIG-AP (Roche) at $4{ }^{\circ} \mathrm{C}$ overnight. On the third day, INT/BCIP was used for colour detection, and images were acquired with a stereomicroscope (Olympus SZX16).

For immunolabelling, embryos were fixed in $4 \%$ paraformaldehyde and embedded in OCT. Cryostat sections were cut at $8 \mu \mathrm{m}$ in thickness and labelled with primary antibodies overnight at $4{ }^{\circ} \mathrm{C}$, which was followed by incubation with appropriate secondary antibodies: horseradish peroxidase (HRP)-conjugated goat anti-rabbit IgG antibody (1:500, AQ132P; Sigma-Aldrich) or Alexa Fluor 594 goat anti-rabbit IgG secondary antibody (1:500, A11037; Invitrogen). The primary antibodies were used as follows: rabbit anti-HuC (1:200, GTX128365; GeneTex) and rabbit anti-PHH3 (1:200, \#3377; Cell Signaling Technology). Images were acquired with a fluorescence microscope (Olympus IX71) after immunostaining.

\section{Western blotting}

Zebrafish embryos were homogenized in RIPA buffer with protease and phosphatase inhibitors (Beyotime Institute of Biotechnology, Shanghai, China). The supernatants were collected by centrifuging at $12000 \mathrm{rpm}$ at $4{ }^{\circ} \mathrm{C}$ for $15 \mathrm{~min}$. The supernatants were mixed with loading buffer, and equal amounts of protein were loaded onto $15 \%$ SDS-PAGE gels. When the separated proteins were transferred to the PVDF membranes, the PVDFs were incubated with primary antibodies and then with an HRP-conjugated goat anti-rabbit IgG antibody (1:5000, AQ132P; Sigma-Aldrich). The primary antibodies were used as follows: rabbit anti-H3 (1:1000, \#4499; Cell Signaling Technology), rabbit anti-acetyl-H3 (1:1000, \#06-599; Millipore), rabbit anti-H3K27me3 (1:1000, \#9733; Cell Signaling Technology), rabbit anti-H3K9me3 (1:1000, \# 13969; Cell Signaling Technology), rabbit anti-GFP (1:1000, \#2956; Cell Signaling Technology), and rabbit anti- $\beta$-actin (1:2000, SAB2100037; Sigma- 

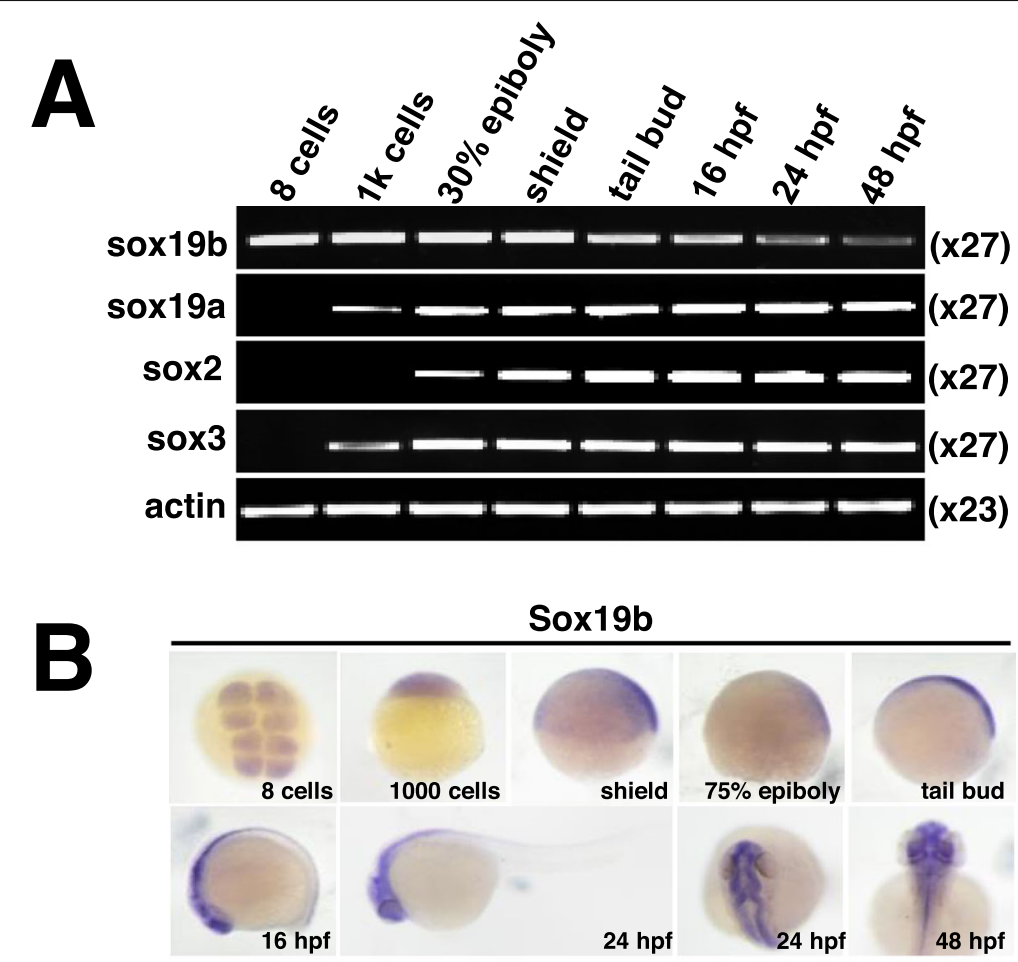

Sox19a

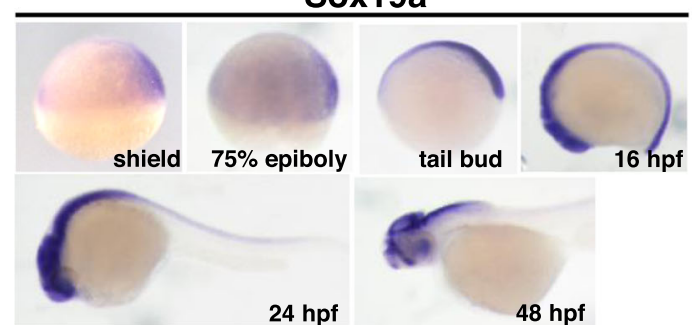

Fig. 1 Expression of the SoxB1 family of genes during zebrafish embryonic development. a RT-PCR was used to analyse the expression level of the SoxB1 family of genes during zebrafish developmental stages, ranging from 8 cells to $48 \mathrm{hpf}$. b Expression analysis of Sox19b and Sox19a in zebrafish was determined by whole-mount in situ hybridization at the indicated stages (from 8 cells to 48 hpf)

Aldrich). Protein bands were analysed by densitometry using Quantity One.

\section{Chromatin immunoprecipitation assay}

Chromatin immunoprecipitation (ChIP) was conducted with an EZ-ChIP kit (Merck Millipore) according to the manufacturer's protocol. Briefly, zebrafish embryos were fixed with $1 \%$ formaldehyde for $15 \mathrm{~min}$ to cross-link histones and DNA, and then glycine was added to stop cross-linking. After washing with PBS three times, the cells were resuspended in SDS lysis buffer with protease inhibitors. After acquiring pelleted nuclei, chromatin was sonicated to shear into $200-500 \mathrm{bp}$. The supernatants were immunoprecipitated with an H3K27me3 antibody (CST) or a control antibody (anti-IgG) overnight at
$4{ }^{\circ} \mathrm{C}$ with rotation, which was followed by incubation with protein $\mathrm{G}$ agarose for $1 \mathrm{~h}$. Protein $\mathrm{G}$ agarose antibody/chromatin complexes were collected, washed, and eluted. Then, cross-links were reversed, and DNA was purified and analysed via real-time PCR. The ChIPqPCR primer sequences were as follows: Ngn1 (forward, 5 '-tctcccagcccaccaataag-3'; reverse, 5' -tcacagcttgaggtttccat-3') and ascl1a (forward, 5' -ccattgaagccacacgtgaa- $3^{\prime}$; reverse, $5^{\prime}$-tgaactgctgctggtttacg- $\left.3^{\prime}\right)$.

\section{Statistical analysis}

Statistical significance was performed using GraphPad Prism 5.0 Software. Data were calculated using Student's $t$ tests for comparison of the two groups. One-way 
analysis of variance (ANOVA) was performed for three or more groups (as in Fig. 5b, c), followed by the LSD post hoc test. Dunnett's T3 test was used when equal variances were not assumed. All bar graphs were plotted as the mean \pm standard error of the mean (SEM), and significance was set at $P<0.05$.

\section{Results \\ Expression pattern of Sox $19 \mathrm{~b}$ during neuronal development in zebrafish}

In this study, zebrafish were selected as an animal model. We first explored the spatiotemporal expression characteristics of the SoxB1 family during zebrafish development by RT-PCR and in situ hybridization. Our results showed that the expression of SoxB1 mRNA in different stages was as follows: Sox $19 b$ was highly expressed during the maternal phase, Sox3 and Sox19a were expressed initially in the 1000 cell phase, and Sox 2 was expressed at the beginning of the $30 \%$ epiboly phase. All four continued to be expressed at 48 hpf (Fig. 1a). With the development of the zebrafish embryos, the distribution of Sox $19 a$ and Sox $19 b$ became gradually concentrated in the neural tube region, especially from the tailbud stage to $48 \mathrm{hpf}$ (Fig. 1b). The maternal expression of Sox $19 \mathrm{~b}$ and the specific neural tube expression, which is similar to Sox2 in mammals, have attracted our attention and emphasize the importance of Sox19b in neural tube development.

\section{Knockdown of Sox19b caused deficits in the development of neural tubes}

To verify the role of Sox19b during zebrafish embryonic development, we disrupted its translation using antisense morpholino (MO) oligonucleotides, and the specificity and efficiency of the treatment were assessed. Western blot confirmed that the MO oligonucleotides inhibit the translation of Sox19b mRNA (Fig. 2a). We analysed the phenotype and found that zebrafish embryos appeared as follows: the telencephalon and diencephalon were diminished or even lost, the body axis became shorter, and the tail turned up after knockdown of sox19b (Fig. 2b, c). To gain further insights into the molecular mechanism behind this phenotype, we analysed the expression of several markers during neural tube formation. The results showed that the expression levels of both otx 2 (anterior neuroectoderm) and pax2 (neural ectoderm) were significantly decreased compared with the control group (Fig. 2d). H\&E staining of embryo sections at 24 hpf confirmed that the neural tube in the Sox19b MO group became thinner, the lumen was enlarged, and the number of epithelial cells decreased in the forebrain and spinal cord (Fig. 3a).

\section{Knockdown of Sox19b inhibited the proliferation and induced premature differentiation of NSCs}

The proliferation, differentiation, and migration of NSCs are the cytological basis for the normal development of neural tubes. A decrease in the number of epithelial cells means that the proliferation and differentiation of NSCs may be affected. We first detected the effect of Sox19b on the proliferation of NSCs with BrdU incorporation assays. The results showed that the number of BrdUpositive cells was decreased in the Sox19b MO group (Fig. 3b). Moreover, we found that the level of PCNA, a well-accepted marker of proliferation, was significantly reduced in the Sox19b MO group (Fig. 3c). We then explored the effect of Sox $19 \mathrm{~b}$ on the differentiation of NSCs. Immunohistochemical staining for $\mathrm{HuC}$ showed expression in early neuronal cells, and we found that the absolute quantity of $\mathrm{HuC}$-positive cells decreased. Surprisingly, the proportion of early neuronal cells $\left(\mathrm{HuC}^{+}\right.$ cells/total cells) increased (Fig. 3d), which suggests that the fate of NSCs in the neural tube was abnormal. Taken together, these results suggested that the neural tube defects might be due to the impaired proliferation and differentiation of NSCs after injecting the Sox19b MO.

Next, we explored the changes in factors related to the fate of NSC differentiation in zebrafish embryos at a critical stage (10-11 hpf). The results showed that the transcription levels of $\mathrm{Ngn1}$, ascl1a, HuC, and Islet1 were increased, while the transcription level of Her3 was decreased (Fig. 4a, b). Cyclin-dependent kinase (CDK) inhibitors are important for differentiation. We found that, compared with the control group, the expression of the CDK inhibitors $p 21, p 27$, and $p 57$ was increased after injecting of Sox19b MO (Fig. 4c). We used a phospho-histone H3 (p-H3) antibody to label the mitotic cells and found that the number of $\mathrm{p}-\mathrm{H}^{+}$NSCs in the Sox19b MO group was significantly smaller than what was seen in the control group (Fig. 4d). These results showed that NSCs were prematurely differentiated, and the number of undifferentiated NSCs was reduced.

\section{Knockdown of Sox19b inhibited the expression of EZH2 and reduced the trimethylation of histone $\mathrm{H} 3$}

In zebrafish, the regulation of NSCs involves the coordination of multiple signalling pathways, such as FGF, Wnt, and BMP. Among them, FGF is mainly involved in the regulation of NSCs from the end of gastrulation. Our results showed that there was a decrease in the activity of the FGF signalling pathway after knocking down Sox19b, but injection with $f g f 3$ mRNA could not rescue the most obvious phenotype, which was telencephalic loss and premature differentiation of NSCs (Fig. 5a-c). These results suggest that there may be another mechanism by which Sox19b regulates NSCs. 

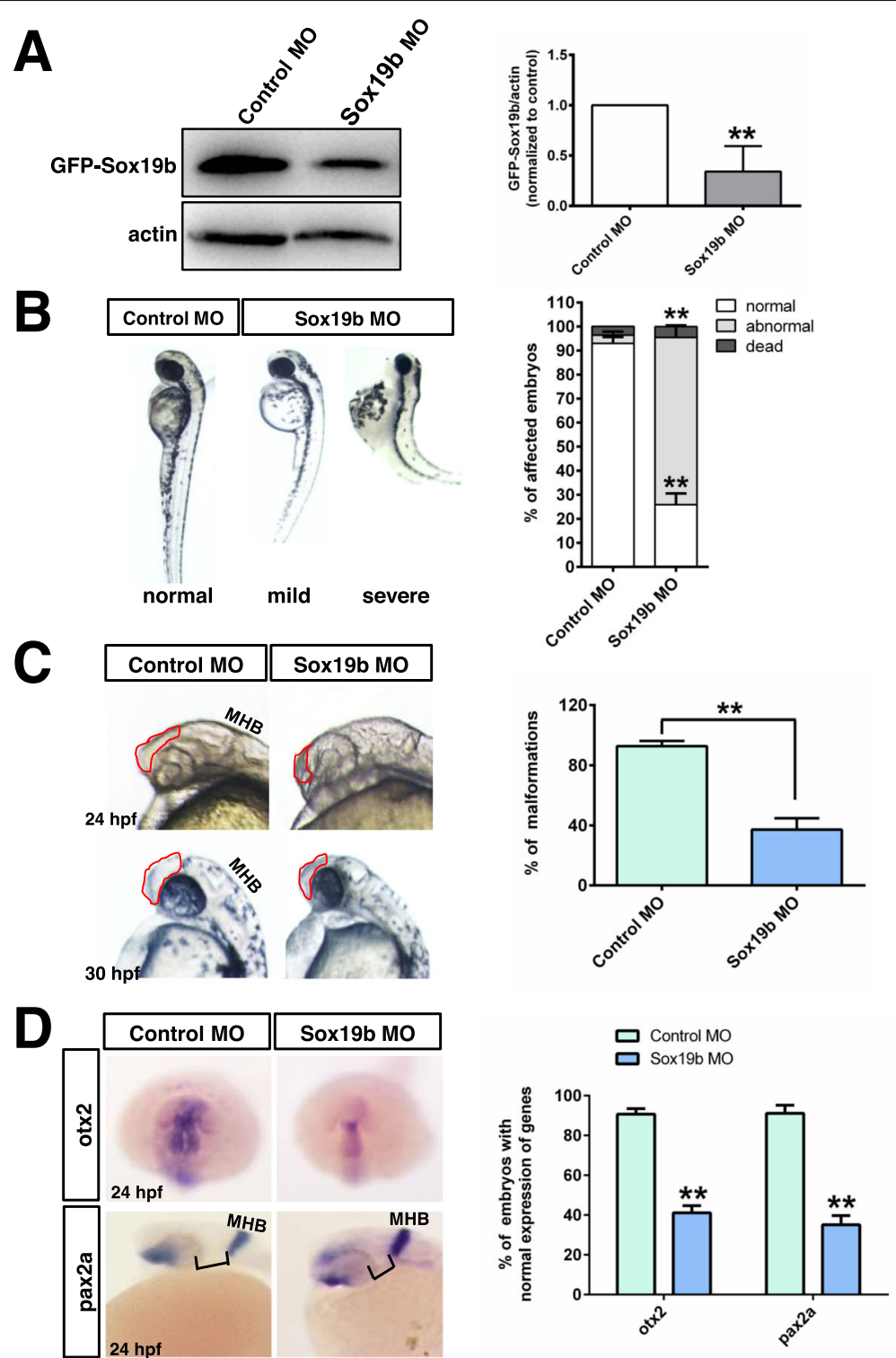

Fig. 2 Zebrafish embryos displayed NTDs after knockdown of Sox19b. a Western blot confirmed that Sox19b MO could interfere Sox19b mRNA translation. $\mathbf{b}$ The neural tube development of zebrafish embryos was abnormal after injection with a Sox 19b MO at 48 hpf. There were two main results. The mild phenotype consisted of a smaller head and shorter body axis. The severe phenotype had a partial head area deletion, microphthalmos, shorter body axis, and tail turned up. Bar graphs show the statistical data for the embryo numbers. Data represent the mean of at least three independent experiments \pm SD. ${ }^{* *} P<0.01$ versus control. c The zebrafish embryos developed to $24 \mathrm{hpf}$ and $30 \mathrm{hpf}$, and the embryos injected with Sox19b MO showed that the area of the telencephalon and diencephalon decreased significantly compared with controls. The bar graphs show the percentage of normal embryos in each group. Data represent the mean of at least three independent experiments \pm SD. ${ }^{* *} P<0.01$ versus control. d Whole-mount in situ hybridization analysis of neuroectoderm marker expression at 24 hpf. The expression of otx 2 and pax2a were decreased in the Sox19b MO group compared with what was observed in the controls. Bar graphs show the percentage of normal embryos in each group. Data represent the mean of at least three independent experiments $\pm S D$. ${ }^{*} P<0.05$ versus control

Histone modifications play vital roles in the regulation of gene transcription and embryonic development. It is well known that histone lysine acetylation can significantly promote gene expression, while histone lysine methylation has more complex effects on gene expression (38-40). We first detected the level of histone acetylation and found that the histone acetylation levels in the Sox19b MO group did not change significantly (Fig. 6a). We further found that the level of H3K27me3 in the Sox19b MO group was significantly decreased, but the change of H3K9me3 was not significant (Fig. 6b). The ChIP-qPCR results showed that the levels of 

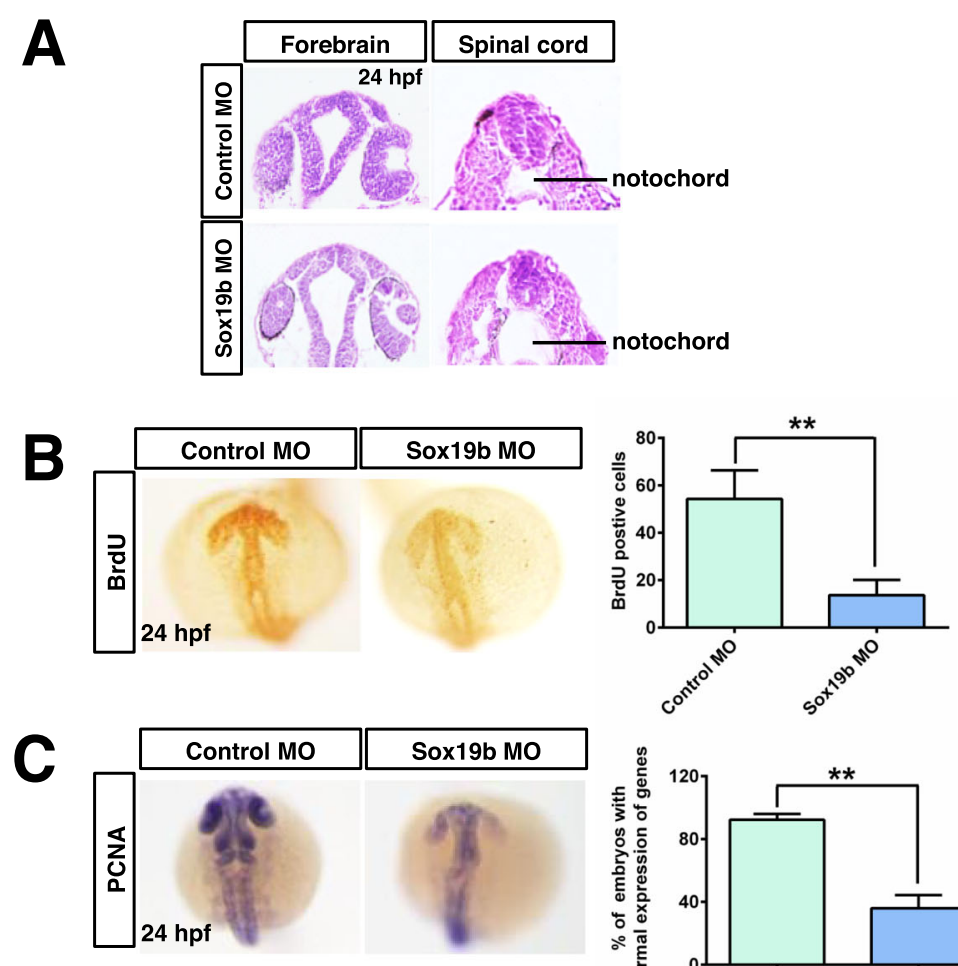

D
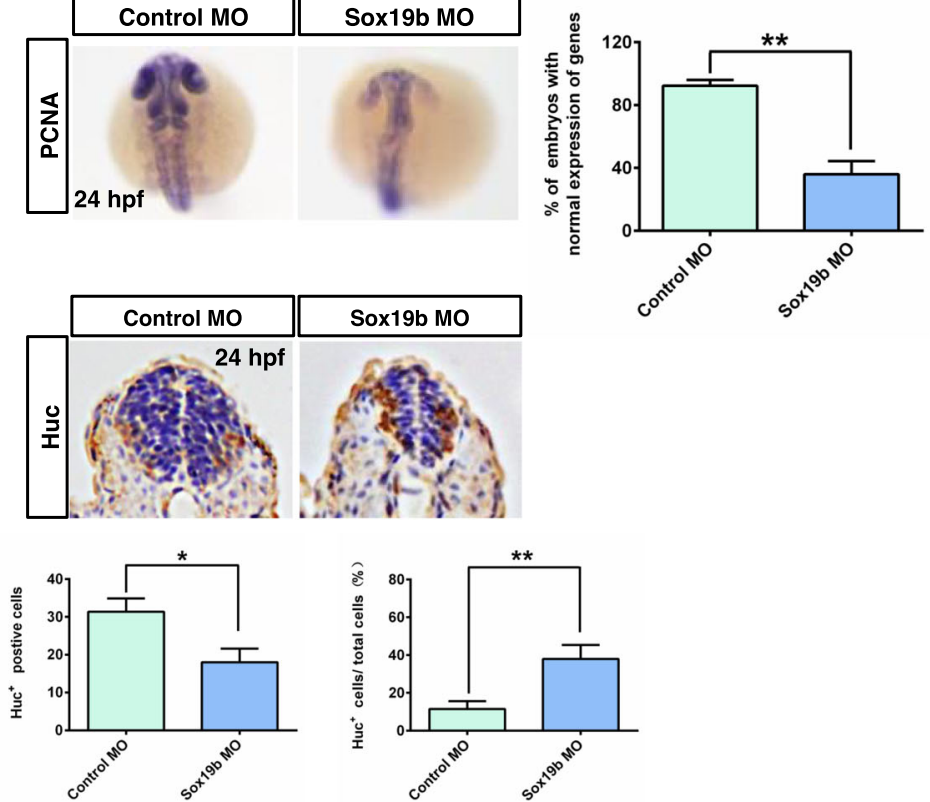

Fig. 3 Effect of Sox19b on NSCs in neural tubes. a H\&E histological sections of the brain and spinal cord of zebrafish embryos at 24 hpf showed that the number of cells and cell layers in the neural tube decreased, and the ventricle expanded in the Sox19b MO-injected embryos compared with controls. b BrdU labelling of control MO- and Sox19b MO-injected embryos at 24 hpf. Data represent the mean of at least three

independent experiments $\pm S D$. ${ }^{* *} P<0.01$ versus control. $\mathbf{c}$ The expression of PCNA was detected by whole-mount in situ hybridization, and the expression was used to detect proliferating cells in zebrafish embryos of control MO MO- and Sox19b MO-injected embryos. $\mathbf{d}$ Immunolabelling of HuC (early neuronal marker) at $24 \mathrm{hpf}$ showed that the expression of HuC was ectopic in Sox 19b morphants, and the expression of HuC appeared in some regions that were enriched with NSCs. Data represent the mean of at least three independent experiments \pm SD. ${ }^{*} P<0.05$ versus control

H3K27me3 at the promoters of Ngn1 and ascl1a were significantly decreased after injection with the Sox19b MO (Fig. 6c, d). It is known that histone methylation levels are controlled by histone methyltransferase (HMT). The level of H3K27me3 is regulated by the H3K27me3 methylase EZH2. We found that the mRNA and protein levels of EZH2 in the Sox19b MO group were significantly decreased compared with what was observed in the control group (Fig. 7a, b). Overall, we concluded that Sox19b may regulate the differentiation of NSCs through EZH2-mediated regulation of H3K27me3 levels.

Taken together, we first discovered the role of Sox19b in NTDs (Fig. 8), established the regulatory network of transcription factors and epigenetic factors to NSCs, and provided new clues and theoretical guidance for the clinical treatment of NTDs. 

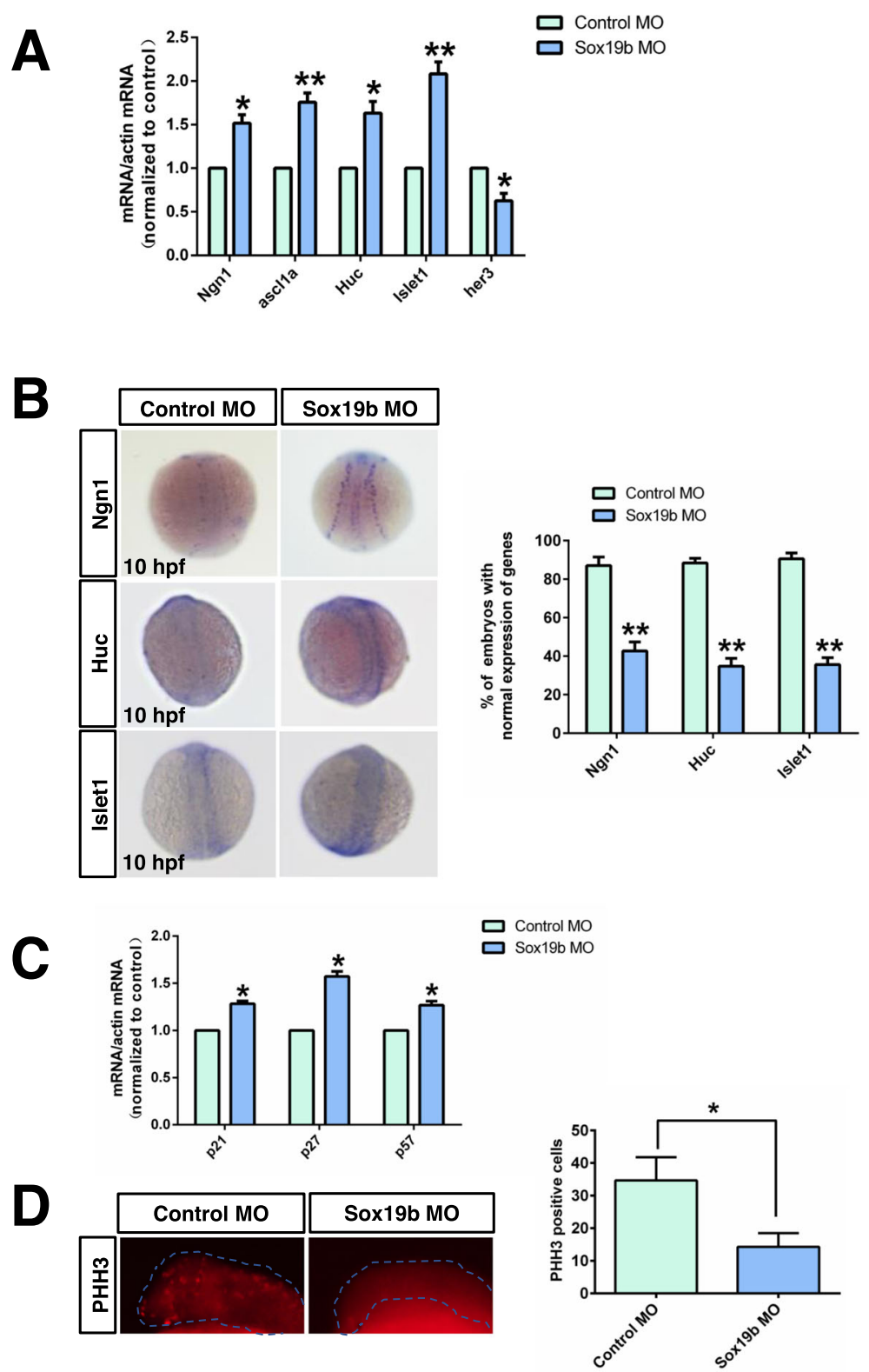

Fig. 4 NSCs in embryos injected with sox19b MO were prematurely differentiated. a Quantitative RT-PCR analysis for Ngn1, ascl1a, HuC, Islet1, and her3. Compared with the control group, the mRNA levels of Ngn1, asclia, HuC, and Islet1 in Sox19b MO-injection embryos increased significantly, but the mRNA level of her3 decreased significantly. $\mathbf{b}$ The expression levels of Ngn1, HuC, and Islet1 were detected by whole-mount in situ hybridization. Bar graphs show the percentage of normal embryos in each group. Data represent the mean of at least three independent experiments \pm SD. ${ }^{*} P<0.05,{ }^{* *} P<0.01$ versus control. c Quantitative RT-PCR analysis is shown for $p 21, p 27$, and $p 57$. Knockdown of Sox19b caused a significant increase in the mRNA levels of cyclin-dependent kinase inhibitor. Data represent the mean of at least three independent experiments \pm SD. ${ }^{*} P<0.05$ versus control. $\mathbf{d}$ Control and Sox $19 \mathrm{~b}$ MO-injected embryos were analysed by immunofluorescence staining of PHH3 (a marker of mitosis, red). Data represent the mean of at least three independent experiments $\pm S D .{ }^{*} P<0.05$ versus control

\section{Discussion}

NTDs are among the most common and serious birth defects in humans. Failure of the embryonic process to generate neural tube closure can lead to serious neurological consequences or even lethality $[3,4]$. The generation, proliferation, differentiation, and migration of NSCs and the resulting morphogenesis are the cytological basis for neural tube development, and abnormalities in this process lead to neural tube defects [9]. Studies uncovering the molecular regulation of NSCs are of major importance for the prevention and treatment of NTDs. In this study, we used zebrafish as an animal model. We found that loss of the SoxB1 family transcription factor Sox19b resulted in deformities 

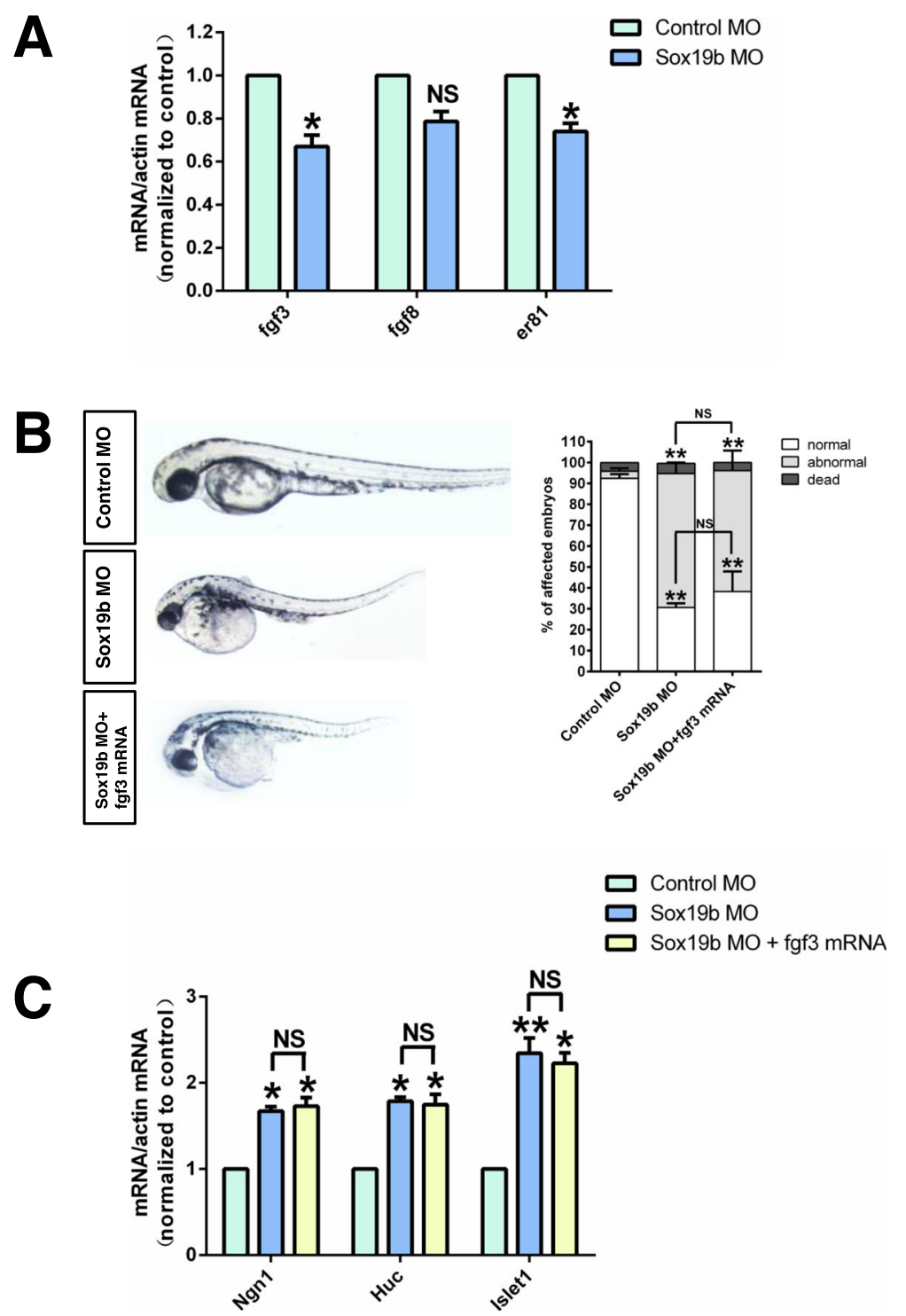

Fig. 5 The FGF pathway did not play the most important role in the Sox19b regulation of NSCs. a Quantitative RT-PCR analysis is shown for fgf3, fgf8, and er81. Compared with the controls, the mRNA levels of fgf3 and er81 in Sox19b MO-injected embryos decreased significantly. Data represent the mean of at least three independent experiments \pm SD. ${ }^{*} P<0.05$ versus control. b Compared with embryos injected with Sox $19 \mathrm{~b}$ MO, the NTD appearance in zebrafish embryos was not rescued by the addition of fgf3 mRNA. c Quantitative RT-PCR analysis for Ngn 1, Huc, and Islet1. Fgf3 mRNA could not change the increase in Ngn1, Huc, or Islet1 induced by Sox19b MO treatment. Data represent the mean of at least three independent experiments $\pm \mathrm{SD} .{ }^{*} P<0.05,{ }^{* *} P<0.01$ versus control; ns, no significant difference

that were similar to neural tube defects in zebrafish embryos. Furthermore, decreased proliferation and increased premature differentiation of NSCs were found in the zebrafish embryo with knocked down Sox19b. This suggests that Sox19b may participate in the development of neural tubes by regulating the transition from proliferation to differentiation and the fate of NSCs. Finally, our results showed that Sox19b regulated the trimethylation of histone H3K27 through EZH2. After inhibition of Sox19b, decreased H3K27me3 levels altered the chromatin state of
Ngn1 and ascl1a and reduced their transcription, thereby affecting the differentiation of NSCs.

The SoxB1 family plays the most important role in the proliferation and differentiation of NSCs [16], and its expression is significantly reduced in patients with NTDs and animal models [17-19]. The SoxB1 family in zebrafish includes Sox1a, Sox1b, Sox2, Sox3, Sox19a, and Sox19b [22]. Early studies on zebrafish SoxB1 have focused on Sox 2 and Sox3. Sox 2 regulates the development of the retina and the lens. Inhibition of Sox2 


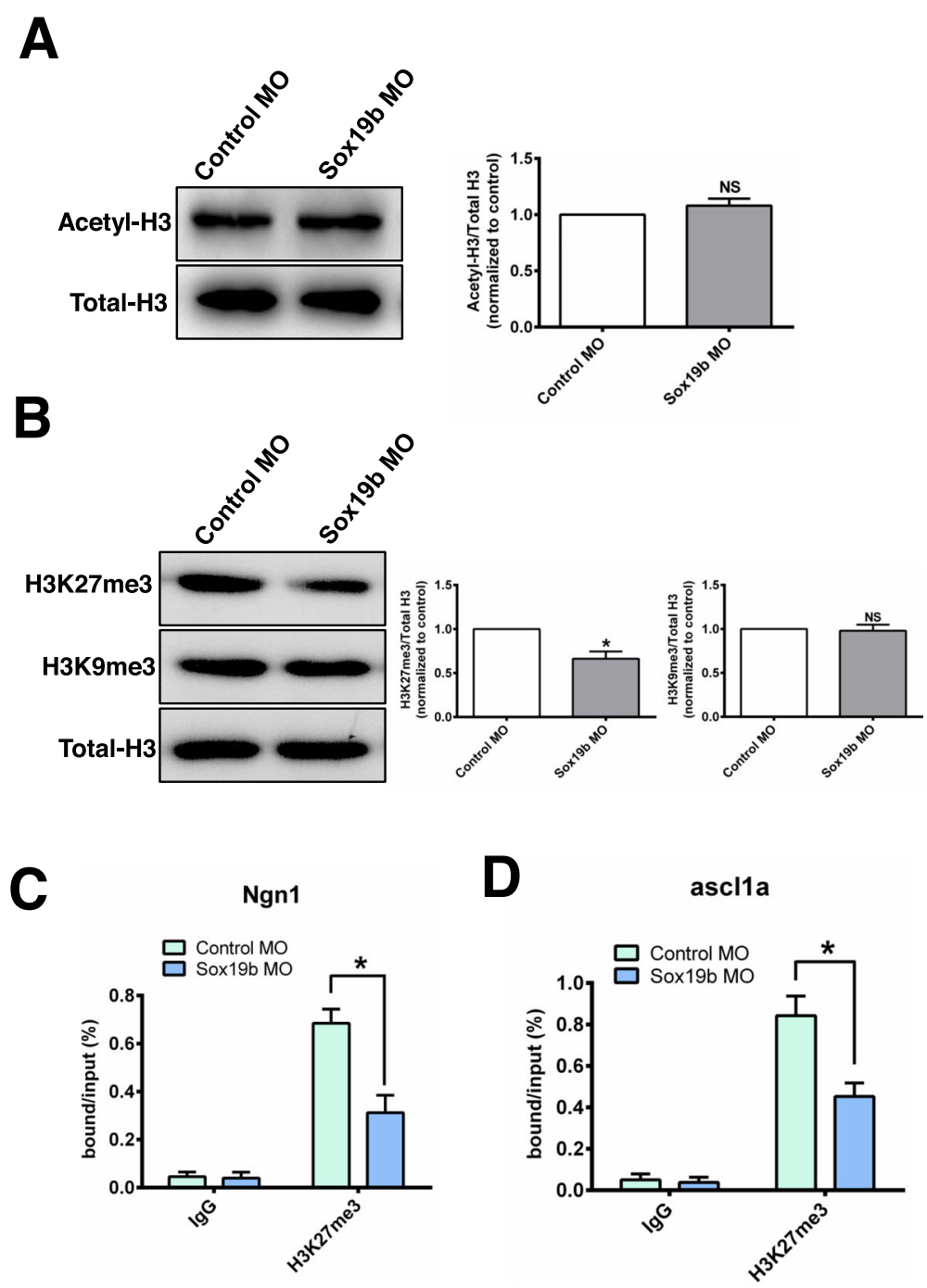

Fig. 6 Sox19b regulated NSCs through an epigenetic mechanism. a Western blot analysis of acetyl-H3 protein levels in control and Sox19b MOinjected embryos. Sox19b had no effect on the acetyl-H3 levels. b Western blot analysis of H3K27me3 and H3K9me3 levels in control and Sox19b MO-injected embryos. The H3K27me3 level decreased after knocking down sox19b, but the level of H3K9me3 remained unchanged. Data represent the mean of at least three independent experiments \pm SD. ${ }^{*} P<0.05$ versus control. c, $\mathbf{d}$ Control and Sox $19 \mathrm{~b}$ MO-injected embryos were immunoprecipitated with anti-H3K27me3 antibody, and the isolated DNA was analysed by using gene-specific ChIP primers. Rabbit IgG was used as a negative control. DNA from each ChIP sample was normalized by the corresponding input sample. Compared with the controls, the level of H3K27me3 in the Ngn1 and asclla promoter regions decreased significantly in Sox19b MO-injected embryos. Data represent the mean of at least three independent experiments $\pm \mathrm{SD} .{ }^{*} P<0.05$ versus control

results in anophthalmos and affects the development of the hypothalamus [24]. Sox3 is mainly involved in the process of neural induction. Overexpression of Sox 3 results in the formation of an extra neural plate [25]. However, it has also been reported that zebrafish embryos had no obvious central nervous system (CNS) dysplasia phenotype after inhibiting the expression of Sox 2 and Sox3 separately [23]. The above studies show that there are still many controversies in the existing research and the role of the SoxB1 family in zebrafish neural tube development.
Sox19a and Sox19b are unique to bony fish [22]. Sox19a and Sox19b have been continually expressed in the region of the presumptive CNS and appear to be the earliest molecular markers of the CNS [22]. Compared with Sox19a, Sox19b is maternally expressed; its transcription starts at gastrulation, and it is expressed in the early stages of the presumptive neuroectoderm. Later, Sox19b is restricted to the presumptive CNS, including the forebrain, midbrain, hindbrain, and spinal cord [22, 26]. Our results also confirm these expression results. Previous studies have shown that overexpression of 

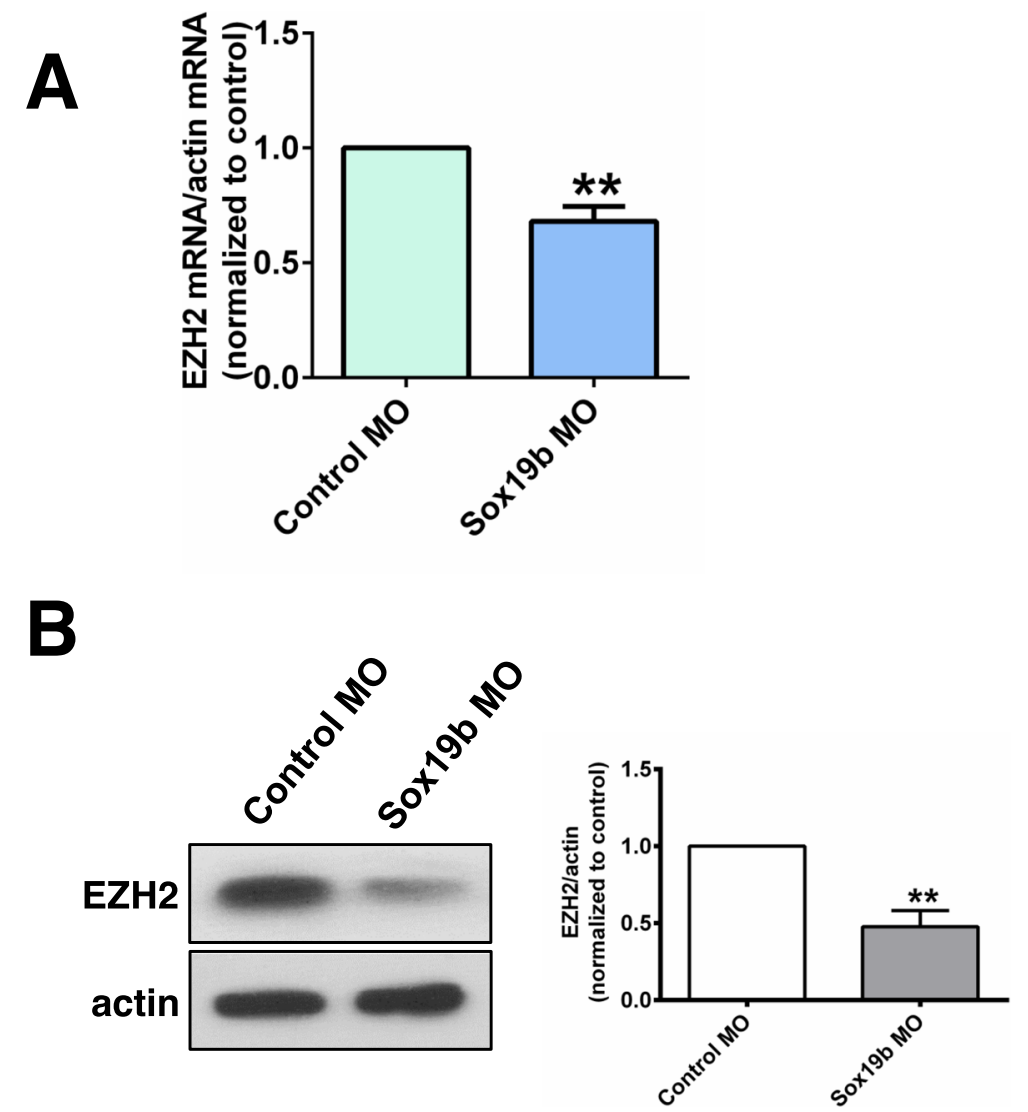

Fig. 7 Sox19b regulated NSCS through EZH2. a Quantitative RT-PCR analysis is shown for EZH2. Compared with the controls, the mRNA level of EZH2 in Sox19b MO-injected embryos decreased significantly. b Western blot analysis of EZH2 protein levels in control and Sox19b MO-injected embryos. The protein level of EZH2 decreased after knocking down of Sox19b. Data represent the mean of at least three independent experiments \pm SD. ${ }^{* *} P<0.01$ versus control

Sox19a and Sox19b could repress the expression of organizer genes, and only Sox19b could effectively rescue the effects of a Sox3 dominant-negative construct [27]. In this study, we used MO oligonucleotides to inhibit the expression of Sox19a and Sox19b. The results showed that after the injection of a Sox19b MO, embryos exhibited an obvious neural tube defect phenotype, but there was no obvious abnormality in the embryo injected with a Sox19a MO (data not shown). These results confirmed the specificity of Sox $19 \mathrm{~b}$ function. Previous studies have shown that Sox19b functions as an essential CNS anteroposterior patterning determinant by regulating organizer activity [26]. Sox19b participates in the regulation of neurogenesis in the spinal cord following an injury $[28,29]$. In this study, we showed that the embryos injected with a Sox19b MO exhibited a neural tube defect phenotype: the telencephalon and diencephalon were diminished or even missing, and the body was shortened. Furthermore, the expression levels of otx2 (anterior neuroectoderm) and pax2a (neural ectoderm) in the Sox19b MO group were also decreased. The morphological analysis found that the number of cells in the neural tube of the Sox19b MO-injected embryos was decreased, and the chamber was enlarged.

The proliferation, differentiation, and migration of NSCs are the cytological basis for the normal development of neural tubes. A decrease in the number of epithelial cells means that the proliferation and differentiation of NSCs may be affected. First, we examined the effect of Sox19b reduction on the proliferation of NSCs. We found that BrdU-positive cells were decreased and that PCNA was also significantly reduced in the Sox19b MO group. Subsequently, the differentiation of NSCs was also confirmed to be abnormal. The results showed that although the absolute quantity of $\mathrm{HuC}$-positive cells decreased, the ratio of neuronal cells $(\mathrm{HuC}+$ cells/total cells) increased. At the time of the formation of the neural plate $(10 \mathrm{hpf})$, the transcription levels of $N g n 1$, asclla, $\mathrm{HuC}$, and Islet1 were increased, but the transcription level of her3 was decreased. These results showed that loss of Sox 19b resulted in the premature differentiation of NSCs. Meanwhile, the expression levels of the CDK inhibitors $p 21, p 27$, and $p 57$ were increased, which further suggested that inhibition of Sox19b led to 


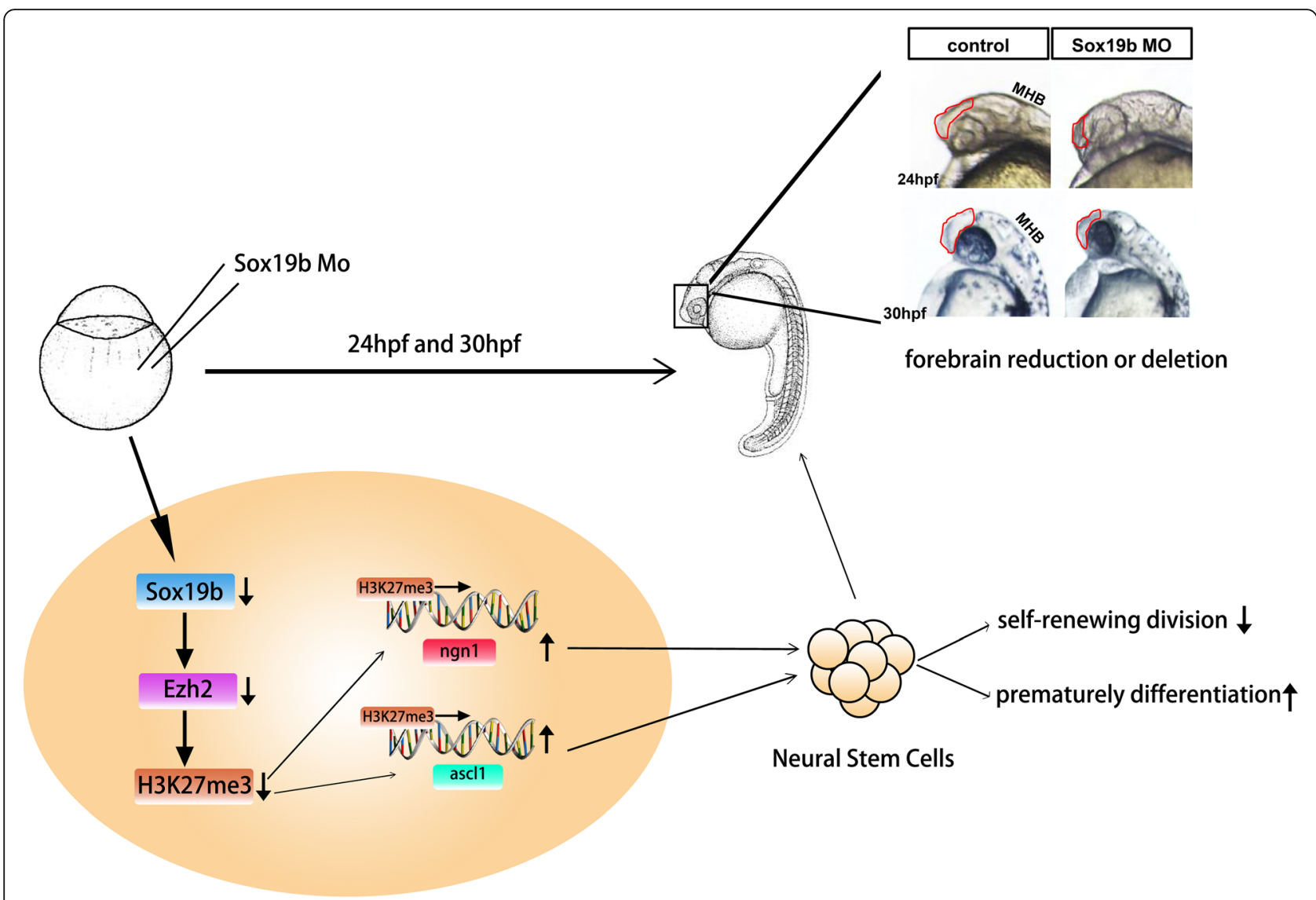

Fig. 8 Schematic representation of Sox19b-regulated neural differentiation of NSCs in zebrafish neural tube development

the premature exit of NSCs from the cell cycle. Our data suggested that Sox19b played an important role in the fate determination of NSCs.

In mammals, Sox 2 promotes the proliferation of NSCs and maintains the number of NSCs by increasing the transcription of Notch1, RBP-J, and Hes5 in the Notch signalling pathway. Sox3 binds directly to the fgf3 promoter region and regulate its transcription [30, 31]. We detected multiple signalling pathways affecting the proliferation and differentiation of NSCs in zebrafish, such as FGF, Wnt, and BMP. We found that the loss of Sox19b affected the FGF signalling pathway, and the expression levels of $f g f 3$ and er81 were significantly decreased. However, injection of $f g f 3$ mRNA could not rescue the NTD phenotype or the premature differentiation of NSCs.

Increasing evidence has shown that transcription factors and epigenetic modifications work together in regulating gene transcription [32,33]. Our team conducted a series of studies confirming that covalent modifications of histones are involved in the regulation of NSC neuronal differentiation [34-36]. Covalent modification of histones mainly includes acetylation, methylation, phosphorylation, and ubiquitination of histone $\mathrm{H} 3$ and H4 [37]. In this study, we found that inhibition of Sox19b did not affect the acetylation of histone H3, but it did alter the trimethylation of histone H3, mainly the trimethylation of lysine site 27. Removal of this repressive chromatin modification results in marked upregulation of gene expression, the consequence of which is a shift in the balance between self-renewal and differentiation towards differentiation.

\section{Conclusions}

Based on these results, we conclude that Sox19b plays a crucial role in neural tube development and regulates the differentiation fate of NSCs. We first found that Sox19b maintained the level of H3K27me3 through $\mathrm{EZH} 2$, ensuring that NSCs would not differentiate prematurely. We have established a regulatory network of transcription factors and epigenetic factors in the fate of NSC differentiation. From the perspective of disease treatment, this study provides a good basis for further revealing the role of the SoxB1 family in the development of NTDs and provides new ideas and new clues for the clinical treatment of NTDs. 


\section{Abbreviations}

NTDs: Neural tube defects; SoxB1: SRY-related HMG box B1; CNS: Central nervous system; NSCs: Neural stem cells; MO: Morpholino; CDK: Cyclindependent kinase

\section{Acknowledgements}

Not applicable.

\section{Authors' contributions}

$X \mathrm{~L}$ and WJZ designed and performed the majority of the laboratory work and the writing of the manuscript. XYL, MG, and SFJ were involved in the collection and assembly of the data. WYT and GYJ contributed to the data analysis and interpretation. AJH was responsible for the data analysis and interpretation, experimental conception and design, manuscript writing, and financial support. All authors read and approved the final manuscript.

\section{Funding}

This work was supported by funding from the National Natural Science Foundation of China (No. 81571093 and 81771219) and the Natural Science Foundation of Shandong Province (No. 2018GSF118076).

\section{Availability of data and materials}

The datasets generated and/or analysed during the current study are included within the article and are available from the corresponding author on reasonable request.

\section{Ethics approval and consent to participate}

All animal experiments are approved by the National Institutes of Health Guide for the Care and Use of Laboratory and the Institutional Animal Care and Use Committees of Shandong University.

\section{Consent for publication}

Not applicable

\section{Competing interests}

The authors declare that they have no competing interests.

\section{Author details}

'Key Laboratory of the Ministry of Education for Experimental Teratology, Shandong Provincial Key Laboratory of Mental Disorders, Department of Human Anatomy and Histoembryology, School of Basic Medical Sciences, Shandong University, 44\#, Wenhua Xi Road, Jinan 250012, Shandong, China. ${ }^{2}$ Foot and Ankle Surgery Center of Shandong University and Department of Hand and Foot Surgery, The Second Hospital of Shandong University, Jinan, Shandong, China. ${ }^{3}$ National Research Center for Assisted Reproductive Technology and Reproductive Genetics, Center for Reproductive Medicine, Shandong University, Jinan, China.

\section{Received: 1 September 2019 Revised: 29 October 2019} Accepted: 14 November 2019 Published online: 16 December 2019

\section{References}

1. Sadler TW. Embryology of neural tube development. Am J Med Genet C: Semin Med Genet. 2005;135C(1):2-8.

2. Wallingford JB. Neural tube closure and neural tube defects: studies in animal models reveal known knowns and known unknowns. Am J Med Genet C: Semin Med Genet. 2005;135C(1):59-68.

3. Copp AJ, Stanier P, Greene ND. Neural tube defects: recent advances, unsolved questions, and controversies. Lancet Neurol. 2013;12(8):799-810.

4. Moore CA, Li S, Li Z, Hong SX, Gu HQ, Berry RJ, et al. Elevated rates of severe neural tube defects in a high-prevalence area in northern China. Am J Med Genet. 1997;73(2):113-8.

5. Zhu L, Ling H. National Neural Tube Defects Prevention Program in China. Food Nutr Bull. 2008;29(2 Suppl):S196-204.

6. Detrait ER, George TM, Etchevers HC, Gilbert JR, Vekemans M, Speer MC. Human neural tube defects: developmental biology, epidemiology, and genetics. Neurotoxicol Teratol. 2005;27(3):515-24.

7. Copp AJ, Greene ND. Genetics and development of neural tube defects. J Pathol. 2010:220(2):217-30.

8. Greene ND, Copp AJ. Neural tube defects. Annu Rev Neurosci. 2014;37:221-42.
9. Shaker MR, Kim JY, Kim H, Sun W. Identification and characterization of secondary neural tube-derived embryonic neural stem cells in vitro. Stem Cells Dev. 2015;24(10):1171-81.

10. Dhaulakhandi DB, Rohilla S, Rattan KN. Neural tube defects: review of experimental evidence on stem cell therapy and newer treatment options. Fetal Diagn Ther. 2010;28(2):72-8.

11. Junghans D, Herzog S. Cnn3 regulates neural tube morphogenesis and neuronal stem cell properties. FEBS J. 2018;285(2):325-38.

12. Valensisi C, Andrus C, Buckberry S, Doni Jayavelu N, Lund RJ, Lister R, et al. Epigenomic landscapes of hESC-derived neural rosettes: modeling neural tube formation and diseases. Cell Rep. 2017;20(6):1448-62.

13. Pearson BJ, Doe CQ. Specification of temporal identity in the developing nervous system. Annu Rev Cell Dev Biol. 2004;20:619-47.

14. Pinto L, Gotz M. Radial glial cell heterogeneity--the source of diverse progeny in the CNS. Prog Neurobiol. 2007;83(1):2-23.

15. Pevny L, Placzek M. SOX genes and neural progenitor identity. Curr Opin Neurobiol. 2005;15(1):7-13.

16. Bylund M, Andersson E, Novitch BG, Muhr J. Vertebrate neurogenesis is counteracted by Sox1-3 activity. Nat Neurosci. 2003;6(11):1162-8.

17. Kelberman D, de Castro SC, Huang S, Crolla JA, Palmer R, Gregory JW, et al. SOX2 plays a critical role in the pituitary, forebrain, and eye during human embryonic development. J Clin Endocrinol Metab. 2008;93(5):1865-73.

18. Kelberman D, Rizzoti K, Avilion A, Bitner-Glindzicz M, Cianfarani S, Collins J, et al. Mutations within Sox2/SOX2 are associated with abnormalities in the hypothalamo-pituitary-gonadal axis in mice and humans. J Clin Invest. 2006; 116(9):2442-55

19. Saxena AK, Pandey S, Pandey LK. Genetic diversity of stem cells and their functional impact on the development of neural tube defects in Eastern population of India. Genet Mol Res. 2013;12(3):2380-90.

20. Schmidt R, Strahle U, Scholpp S. Neurogenesis in zebrafish - from embryo to adult. Neural Dev. 2013;8:3.

21. Kaslin J, Kroehne V, Benato F, Argenton F, Brand M. Development and specification of cerebellar stem and progenitor cells in zebrafish: from embryo to adult. Neural Dev. 2013;8:9.

22. Okuda Y, Yoda H, Uchikawa M, Furutani-Seiki M, Takeda H, Kondoh H, et al. Comparative genomic and expression analysis of group B1 sox genes in zebrafish indicates their diversification during vertebrate evolution. Dev Dynamics. 2006:235(3):811-25.

23. Okuda Y, Ogura E, Kondoh H, Kamachi Y. B1 SOX coordinate cell specification with patterning and morphogenesis in the early zebrafish embryo. PLoS Genet. 2010;6(5):e1000936.

24. Pavlou S, Astell K, Kasioulis I, Gakovic M, Baldock R, van Heyningen V, et al. Pleiotropic effects of Sox2 during the development of the zebrafish epithalamus. PLoS One. 2014;9(1):e87546.

25. Dee CT, Hirst CS, Shih YH, Tripathi VB, Patient RK, Scotting PJ. Sox3 regulates both neural fate and differentiation in the zebrafish ectoderm. Dev Biol. 2008;320(1):289-301.

26. Hu S, Wu Z, Yan Y, Li Y. Sox31 is involved in central nervous system anteroposterior regionalization through regulating the organizer activity in zebrafish. Acta Biochim Biophys Sin. 2011;43(5):387-99.

27. Shih YH, Kuo CL, Hirst CS, Dee CT, Liu YR, Laghari ZA, et al. SoxB1 transcription factors restrict organizer gene expression by repressing multiple events downstream of Wnt signalling. Development. 2010;137(16):2671-81.

28. Ghosh S, Hui SP. Regeneration of zebrafish CNS: adult neurogenesis. Neural plasticity. 2016;2016:5815439.

29. Hui SP, Sengupta D, Lee SG, Sen T, Kundu S, Mathavan S, et al. Genome wide expression profiling during spinal cord regeneration identifies comprehensive cellular responses in zebrafish. PLoS One. 2014;9(1):e84212.

30. Kuo CL, Lam CM, Hewitt JE, Scotting PJ. Formation of the embryonic organizer is restricted by the competitive influences of Fgf signaling and the SoxB1 transcription factors. PLoS One. 2013;8(2):e57698.

31. Bani-Yaghoub M, Tremblay RG, Lei JX, Zhang D, Zurakowski B, Sandhu JK, et al. Role of Sox2 in the development of the mouse neocortex. Dev Biol. 2006;295(1):52-66.

32. Cimadamore F, Amador-Arjona A, Chen C, Huang CT, Terskikh AV. SOX2LIN28/let-7 pathway regulates proliferation and neurogenesis in neural precursors. Proc Natl Acad Sci U S A. 2013;110(32):E3017-26.

33. He J, Shen L, Wan M, Taranova O, Wu H, Zhang Y. Kdm2b maintains murine embryonic stem cell status by recruiting PRC1 complex to CpG islands of developmental genes. Nat Cell Biol. 2013;15(4):373-84. 
34. Li X, Chen X, Zhou W, Ji S, Li X, Li G, et al. Effect of melatonin on neuronal differentiation requires CBP/p300-mediated acetylation of histone $\mathrm{H} 3$ lysine 14. Neuroscience. 2017;364:45-59.

35. Chen X, Du Z, Shi W, Wang C, Yang Y, Wang F, et al. 2-Bromopalmitate modulates neuronal differentiation through the regulation of histone acetylation. Stem Cell Res. 2014;12(2):481-91.

36. Chen X, Du Z, Li X, Wang L, Wang F, Shi W, et al. Protein palmitoylation regulates neural stem cell differentiation by modulation of EID1 activity. Mol Neurobiol. 2016;53(8):5722-36.

37. Berger SL. The complex language of chromatin regulation during transcription. Nature. 2007:447(7143):407-12.

\section{Publisher's Note}

Springer Nature remains neutral with regard to jurisdictional claims in published maps and institutional affiliations.

Ready to submit your research? Choose BMC and benefit from:

- fast, convenient online submission

- thorough peer review by experienced researchers in your field

- rapid publication on acceptance

- support for research data, including large and complex data types

- gold Open Access which fosters wider collaboration and increased citations

- maximum visibility for your research: over $100 \mathrm{M}$ website views per year

At BMC, research is always in progress.

Learn more biomedcentral.com/submissions 